\title{
Integración de un sistema de información geográfica en la planificación y gestión de los sistemas de distribución eléctrica
}

\author{
Integration of a geographical information system in the planning \\ and management of power distribution
}

\author{
Raúl Sanhueza Hormazábal ${ }^{1} \quad$ Moisés Estrada Ramírez ${ }^{1}$ \\ Recibido 15 de mayo de 2013, aceptado 12 de noviembre de 2013 \\ Received: May 15, 2013 Accepted: November 12, 2013
}

\begin{abstract}
RESUMEN
Este artículo presenta el modelamiento de una red de distribución eléctrica utilizando la tecnología de los sistemas de información geográfica (GIS). Se indica la manera de crear la base de datos necesaria para, mediante un indicador común, asociar la información geográfica con los objetos gráficos de un mapa digital. Como aplicación se presenta el comportamiento de la red frente a un crecimiento previsto de la demanda. El alimentador es real y la cartografía corresponde a la ciudad de Arica, Chile.
\end{abstract}

Palabras clave: Sistemas de información geográfica, redes eléctricas de distribución, flujo de potencia, mapa digital, cartografía.

\section{ABSTRACT}

This article presents the modeling of a power distribution system using geographic information system (GIS) technology. It shows how to create the required database to associate the geographic information with graphic objects in a digital map, through a common indicator. As an application, the document presents the behavior of the network faced with a projected demand growth. The feeder is real and the cartography corresponds to the city of Arica, Chile.

Keywords: Geographic information systems, electric distribution network, power flow, digital map, cartography.

\section{INTRODUCCIÓN}

Las herramientas de simulación se han utilizado en forma extensa durante muchos años en las tareas de planificación y de operación de los sistemas de distribución eléctrica. En estas simulaciones se han estudiado y propuesto un gran número de métodos analíticos, sin embargo, en su naturaleza, ninguno de estos métodos son geoespaciales, a pesar de que el sistema físico que modelan sí lo es.

La información de los sistemas se encontraba en planos y en documentos de texto que no siempre estaban disponibles o actualizados. El advenimiento de los programas de diseño ayudó a superar los problemas que afectaban a los mapas físicos. Aun así, los primeros mapas CAD eran dibujos poco inteligentes, con ninguna capacidad inherente acerca de la conectividad, ni un atributo descriptivo o cuantitativo para la propiedad o las instalaciones representadas sobre el mapa [12].

En el estudio de un sistema de distribución es posible realizar el análisis de varios escenarios para responder preguntas específicas de la red, sin embargo, no se puede mostrar la forma en que el

1 Escuela Universitaria de Ingeniería Eléctrica-Electrónica. Universidad de Tarapacá. Casilla 6-D. 18 de Septiembre 2222. Arica, Chile.E-mail: sanhueza@uta.cl; m.estradaramirez@gmail.com 
sistema se relaciona con su ambiente, es decir, no es posible incluir consideraciones que no tienen relación directa con los cálculos $[2,15,12]$.

Teniendo en cuenta que las redes eléctricas se distribuyen de manera física y geográfica, es práctico presentar los resultados de los estudios que en ella se realizan mediante un despliegue de mapas $[2,1]$. Hoy en día, las empresas eléctricas hacen uso de los sistemas de información geográfico para cubrir sus necesidades tanto de mapeo como de manejo de recursos $[4,8,11,13,18]$.

La metodología GIS permite la gestión de la información espacial. El sistema permite separar la información en diferentes capas temáticas y las almacena independientemente, permitiendo trabajar con ellas de manera rápida y sencilla (Figura 1). Los objetos tienen una relación y comportamiento, asimismo, tienen información asociada (técnica, comercial, registros históricos) que es almacenada en una base de datos [4].

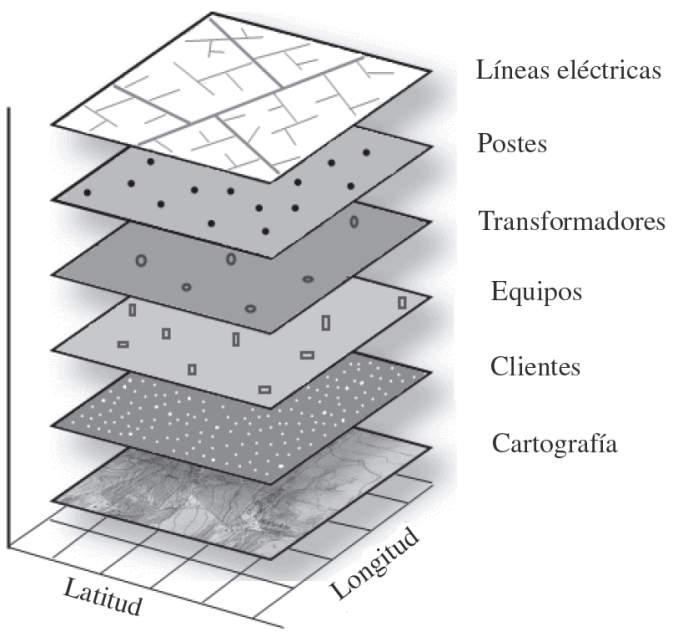

Figura 1. Modelado de una red eléctrica en capas temáticas.

Sin embargo, GIS es más que documentar y mapear la red, pues es posible hacerlo parte de cada una de las áreas de la empresa, pudiendo tener aplicaciones direccionadas al área comercial, mantenimiento de redes, calidad de suministro, cálculo del valor de las instalaciones (VNR), operaciones, ingeniería, planificación, control de flota, entre otros $[9,8,17]$.

En lo que respecta a la planificación, la previsión de la demanda es un estudio que cada año desarrollan las empresas de distribución. Este estudio se lleva a cabo en el sistema de media tensión (MT), de manera desagregada por alimentador y por barra. Con esta información las compañías desarrollan un plan de inversiones, que sustenta un análisis técnico-económico que satisface el crecimiento de la demanda, los estándares de calidad de servicio y la optimización técnico-económica de las redes de MT [5].

La previsión de la demanda responde a la necesidad de elaborar un plan de desarrollo del sistema de distribución, que evalúe alternativas para enfrentar el crecimiento esperado de la demanda, que sea coherente con la capacidad de abastecimiento del proveedor, que asegure una calidad del suministro acorde con las exigencias de la ley eléctrica y de su reglamento [3], y que entregue una rentabilidad aceptable para la empresa, teniendo en cuenta el actual marco regulatorio [6].

El alcance de este trabajo aplica al modelamiento de un sistema eléctrico de distribución dentro del entorno GIS, de manera que en la planeación del sistema se pueda visualizar espacialmente el crecimiento de la demanda experimentado, mostrando, de esta manera, cómo esta tecnología puede ser parte en la toma de decisiones, por ejemplo, para las inversiones que la compañía eléctrica requiera realizar.

\section{MODELO DE INTEGRACIÓN GIS}

Lo que se describe en esta sección no se adhiere a ninguna norma o regla para el diseño de una plataforma GIS. Se indica la manera de cómo construir una estructura de base de datos relacional, con la capacidad de importar/exportar, requerida para el éxito de una gran aplicación. Una de las fortalezas de este formato reside precisamente en la amplia disponibilidad de herramientas ofrecidas para construir datos GIS.

\section{Sistema de prueba}

Para la construcción del modelo en GIS se considera la red eléctrica de prueba indicada en Figura 2.

El sistema corresponde a una red trifásica aérea radial, operando a un voltaje trifásico de 13,8 $\mathrm{kV}$ y capacidad nominal de $16.000 \mathrm{kVA}$. Otras características son: 
- $\quad 34$ km de longitud de línea (en 2, 6 y 8 AWG)

- 220 nudos

- 24 cargas concentradas y equilibradas (representan 24 transformadores)

- $\quad 3.725 \mathrm{kVA}$ de carga conectadas

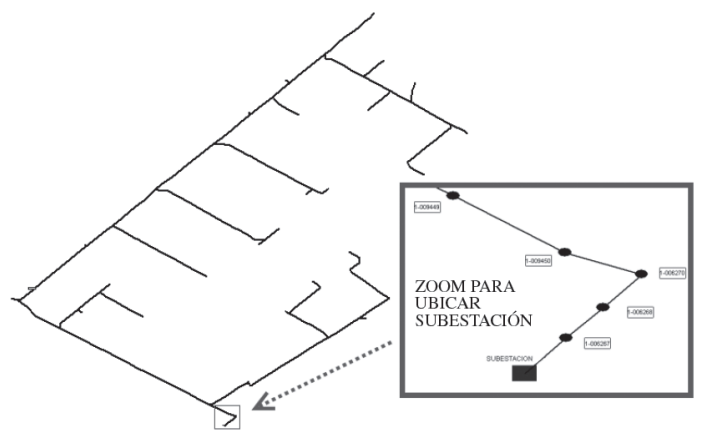

Figura 2. Diagrama unifilar del alimentador de prueba.

En la Tabla 1 se muestra la potencia y demanda por cada transformador, donde la demanda registrada corresponde a un promedio de datos medidos para un periodo de un año.

Tabla 1. Potencia y demanda por transformador (caso base).

\begin{tabular}{|c|c|c|c|}
\hline Transformador & $\begin{array}{c}\text { Potencia } \\
\text { [kVA] }\end{array}$ & $\begin{array}{c}\text { Energía } \\
{[\mathrm{kWh}]}\end{array}$ & Nudo \\
\hline 1 & 150 & 33.486 & $1-007899$ \\
\hline 2 & 150 & 34.236 & $1-007931$ \\
\hline 3 & 150 & 74.003 & $1-007622$ \\
\hline 4 & 200 & 50.546 & $1-007471$ \\
\hline 5 & 150 & 45.232 & $1-007206$ \\
\hline 6 & 150 & 47.365 & $1-007376$ \\
\hline 7 & 250 & 74.431 & $1-007975$ \\
\hline 8 & 100 & 36.161 & $1-007968$ \\
\hline 9 & 150 & 41.653 & $1-007794$ \\
\hline 10 & 150 & 78.315 & $1-008328$ \\
\hline 11 & 150 & 39.429 & $1-008281$ \\
\hline 12 & 75 & 22.026 & $1-008225$ \\
\hline 13 & 75 & 34.285 & $1-008408$ \\
\hline 14 & 150 & 49.673 & $1-008160$ \\
\hline 15 & 75 & 25.562 & $1-007371$ \\
\hline 16 & 75 & 30.255 & $1-009443$ \\
\hline 17 & 75 & 23.427 & $1-007491$ \\
\hline 18 & 75 & 26.665 & $1-007808$ \\
\hline 19 & 50 & 43.012 & $\mathrm{~s} / \mathrm{n} 1$ \\
\hline 20 & 100 & 42.134 & $\mathrm{~s} / \mathrm{n} 2$ \\
\hline 21 & 1000 & 299.845 & $\mathrm{~s} / \mathrm{n} 0$ \\
\hline 22 & 75 & 35.299 & $1-007925$ \\
\hline 23 & 75 & 19.107 & $1-007905$ \\
\hline 24 & 75 & 29.414 & $1-007157$ \\
\hline
\end{tabular}

\section{Análisis de la red}

El modelo GIS se inicia con el desarrollo de la base de datos del sistema. Esta base de datos recoge toda la información física del sistema, es decir, datos de líneas, cargas, barras, transformadores, etc. Una vez que el sistema se modela correctamente, las herramientas de análisis disponibles permiten una amplia capacidad y variedad de cálculos. El resultado de estos análisis se puede exportar al modelo que se construirá en GIS, ampliando la capacidad de reporte.

Los datos de carga del alimentador se obtienen de factores de distribución, que se aplican a los registros de demanda disponibles. La información de carga es relevante para un posterior estudio de esta red.

\section{Casos de estudio}

Se consideran 13 casos en el estudio, un caso base y 12 escenarios que recrean un crecimiento porcentual de la carga, Tabla 2.

Tabla 2. Crecimiento porcentual de la carga.

\begin{tabular}{|c|c|c|}
\hline Casos & Crecimiento [\%] & Nombre Caso \\
\hline caso base & 0 & caso100 \\
\hline 1 & 20 & caso120 \\
\hline 2 & 50 & caso150 \\
\hline 3 & 75 & caso175 \\
\hline 4 & 100 & caso200 \\
\hline 5 & 125 & caso225 \\
\hline 6 & 150 & caso250 \\
\hline 7 & 175 & caso275 \\
\hline 8 & 200 & caso300 \\
\hline 9 & 250 & caso350 \\
\hline 10 & 300 & caso400 \\
\hline 11 & 350 & caso450 \\
\hline 12 & 400 & caso500 \\
\hline
\end{tabular}

El formato de salida de los reportes de cálculos es .xls.

\section{CREACIÓN DE LA RED DE PRUEBA EN ENTORNO GIS}

Implementar un modelo GIS requiere la utilización de cartografía base, es necesario digitalizar toda la información como un conjunto lógico de capas. Luego, se requiere trabajar con un sistema que maneje mapas de información geográfica. Para esto y dado la zona geográfica de interés de la red, el sistema de coordenadas empleado corresponde a: 
- Proyección: UTM, huso 19 sur

- Datum: Sudamericano de 1969

\section{Construcción del modelo}

Datos espaciales:

El proyecto se divide en dos partes: cartografía y red eléctrica. Ambas partes son trazadas en CAD, para luego ser exportados a GIS. El uso de CAD se prefiere debido a la facilidad para dibujar.

Ya en GIS, luego de ser exportadas, cada una de las capas se convierten al formato de datos vectorial nativo. Este formato almacena la ubicación geométrica y la información de atributos de las entidades geográficas. Estas se representan por medio de puntos, líneas y polígonos.

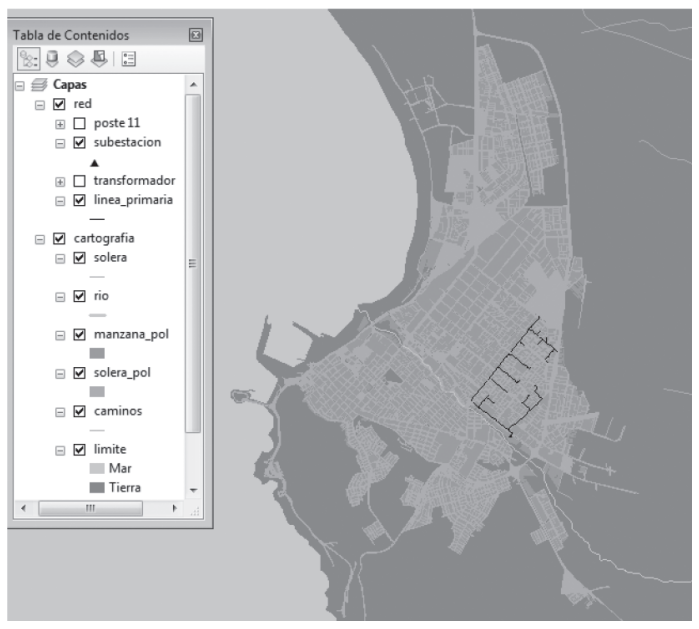

Figura 3. Capas con información cartográfica de la ciudad de Arica.

Para propósitos del trabajo la red trifásica se modeló como una única línea que representa las tres fases (Figura 3, Tabla 3 y Figura 4).

Adicionalmente, se agrega una imagen ráster de la ciudad, la cual define su ubicación por medio de coordenadas de mapa (puntos de control).

\section{Datos de Atributos:}

El segundo paso para construir el modelo GIS es incorporar la información descriptiva desde las hojas de cálculo Excel. Por motivos de operabilidad, los datos descriptivos son exportados desde el formato .xls a dBase. Hecho este paso, y dentro del GIS, los atributos de estas tablas de datos se unen a una capa
Tabla 3. Resumen de capas correspondientes a la red eléctrica.

\begin{tabular}{|l|l|l|}
\hline \multicolumn{1}{|c|}{ Nombre } & Geometría & \multicolumn{1}{c|}{ Descripción } \\
\hline poste 11 & punto & $\begin{array}{l}\text { Poste de 11,5 mts, empleado } \\
\text { en la red MT }\end{array}$ \\
\hline subestación & punto & $\begin{array}{l}\text { Subestación de distribución } \\
(66 / 13,8 \mathrm{kV})\end{array}$ \\
\hline transformador & punto & $\begin{array}{l}\text { Transformadores de } \\
\text { distribución: SE 1 poste } \\
\text { (tipo mochila), SE 2 postes } \\
\text { y SE part para particulares }\end{array}$ \\
\hline línea_primaria & línea & $\begin{array}{l}\text { Alimentador primario } \\
(13,8 \mathrm{kV})\end{array}$ \\
\hline
\end{tabular}

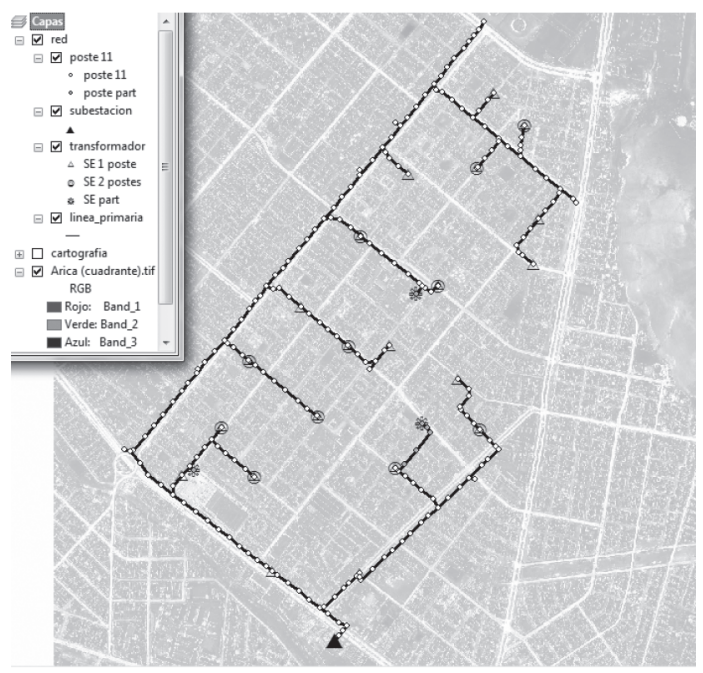

Figura 4. Capas con información del alimentador (vista ampliada sobre una imagen ráster de la ciudad de Arica).

a partir del valor de un campo común existente en ambas tablas, Figura 5.

Los campos "POINT_X" y "POINT_Y", mostrados en Figura 5, corresponden a las coordenadas XY asignadas a las entidades dentro del entorno GIS. Esto entrega valor a cualquiera medición de distancia o coordinación que deseemos realizar con otras capas.

Los pasos para la construcción del modelo se detallan en Figura 6.

El resultado es un conjunto de archivos que comparten la misma referencia espacial; donde sus entidades, independiente del tipo de geometría, se encuentran en un área geográfica común. 


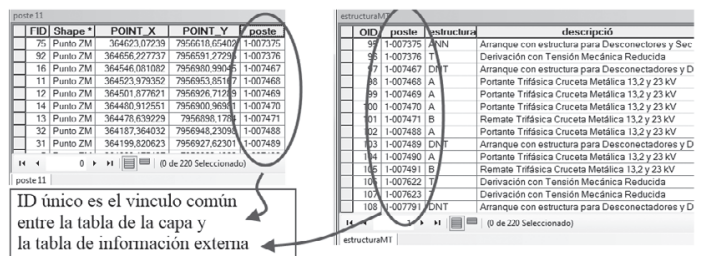

Figura 5. Unión de la tabla de datos "estructuraMT" a la capa "poste 11" a partir del valor de un campo común existente en ambas tablas (poste).

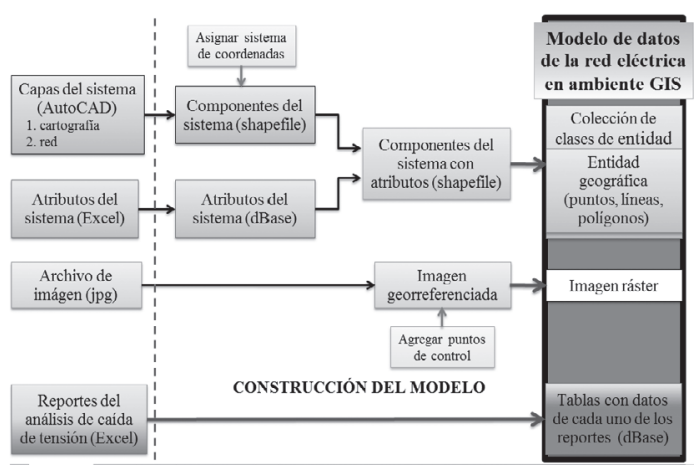

Figura 6. Resumen con pasos de construcción del modelo completo en GIS.

Por último, cada uno de los reportes obtenidos de la simulación de la red se ingresan al modelo GIS, de forma que sean visualizados, consultados y usados en este entorno.

\section{ANÁLISIS DE LA RED DE PRUEBA EN GIS}

Como resultados se extraen los valores de tensión en los diferentes casos estudiados, estos resultados se presentan sobre una grilla que cubre el área de la red. El objetivo es visualizar espacialmente los sectores afectados por la mayor caída de tensión, producto de la variación de carga experimentada en cada caso.

Para ello se construye una herramienta de modelo, representada por la Figura 7. El modelo corresponde a un flujo de trabajo que encadena secuencias de herramientas de geoprocesamiento y suministra la salida de una herramienta a otra herramienta como entrada. En otras palabras, un lenguaje de programación visual que crea flujos de trabajo.

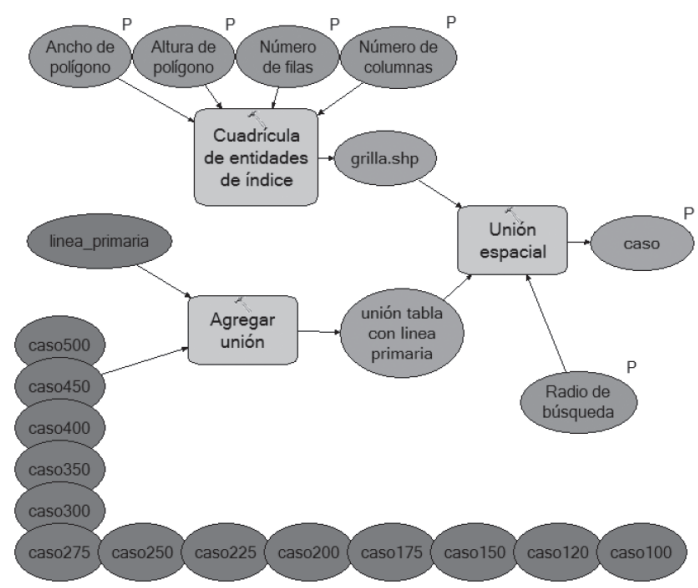

Figura 7. Herramienta con la que se genera una grilla de valores de tensión.

El detalle de la herramienta del modelo, Figura 7, es el siguiente: Los óvalos se consideran variables que pueden ser de entrada o salida. Los rectángulos corresponden a herramientas. Los óvalos, que se acompañan de la letra "P", son parámetros asignados y aparecen en el cuadro de diálogo de la herramienta de modelo final (Figura 8). La herramienta "Cuadrícula de entidades de índice" crea una grilla rectangular, según parámetros. $\mathrm{La}$ herramienta "Agregar unión" vincula los reportes de casos, caso500, caso450,..., o caso100, a la capa línea_primaria, de acuerdo al campo en común "Id_Tramo". La herramienta "Unión espacial" transfiere los atributos de la entidad "unión tabla con línea primaria" a la entidad de destino "grilla. shp", utilizando un radio de búsqueda asignado de $200 \mathrm{~m}$.

\begin{tabular}{|c|c|c|}
\hline Ancho de polígono (opcional) & & \\
\hline 50 & Metros & $\checkmark$ \\
\hline \multicolumn{3}{|l|}{ Altura de polígono (opcional) } \\
\hline 50 & Metros & $\checkmark$ \\
\hline \multicolumn{3}{|l|}{ Número de filas (opcional) } \\
\hline & & 48 \\
\hline \multicolumn{3}{|l|}{ Número de columnas (opcional) } \\
\hline & & 40 \\
\hline \multicolumn{3}{|l|}{ caso } \\
\hline C: |tesis Yresultados|caso.shp & & $E^{+}$ \\
\hline \multicolumn{3}{|l|}{ Radio de búsqueda (opcional) } \\
\hline 200 & Metros & - \\
\hline
\end{tabular}

Figura 8. Cuadro de diálogo de la herramienta "Crea grilla según valores de voltaje". 
La Figura 8 presenta el cuadro para ingresar los parámetros de la herramienta. El modelo se ejecuta y la salida se agrega a la visualización como una nueva capa. El resultado dice así: cualquier cuadro de la grilla que se encuentra dentro de un radio de $200 \mathrm{~m}$ de un tramo del alimentador, y este tramo resulta ser el más cercano a ese cuadro, se transfiere el valor de tensión de dicho tramo a este cuadro.

De la Figura 7 y la Figura 8, con la herramienta "Cuadrícula de entidades de índice", se genera una grilla de 2400x2000 m, con cuadros de 50x50 m, obteniendo la siguiente figura:

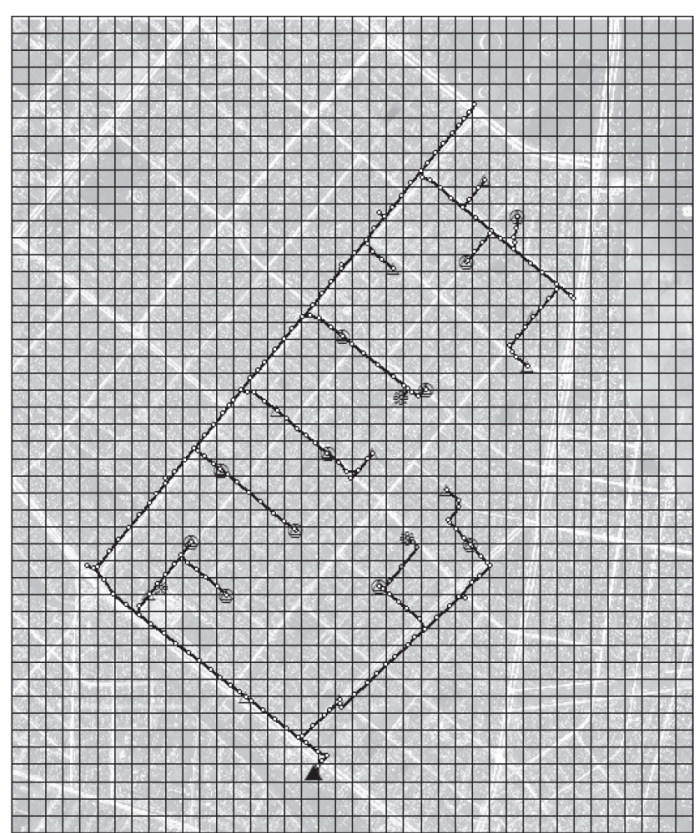

Figura 9. Grilla de $2400 \times 2400 \mathrm{~m}$ con cuadros de $50 \times 50 \mathrm{~m}$, generada con la herramienta "Cuadrícula de entidades de índice".

La unión espacial de esta grilla con los registros de tensión, que contienen los distintos casos, genera capas como las que se muestran en la Figura $10 \mathrm{y}$ Figura 11.

La simbología empleada considera una rampa de colores donde el color rojo que está en la parte superior representa los valores porcentuales de voltaje más bajos, y donde el color verde que está en la parte inferior representa los valores porcentuales de voltaje más cercanos al $100 \%$.
Este trabajo consideró que las caídas de tensión de la subestación no deben superar el 3\%.

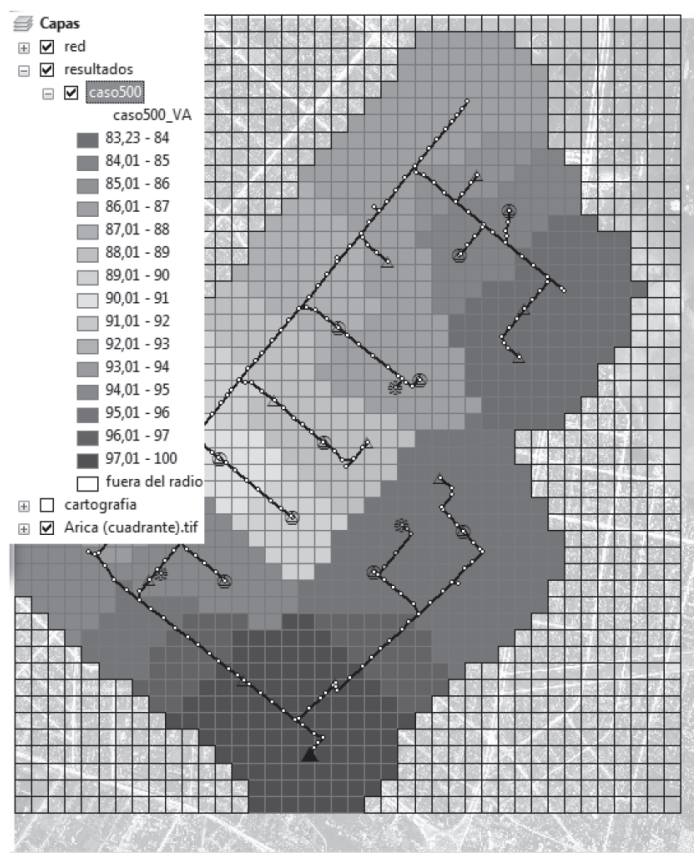

Figura 10. Grilla de valores de voltajes para el "caso500" generada de la herramienta "Crea grilla según valores de voltaje".

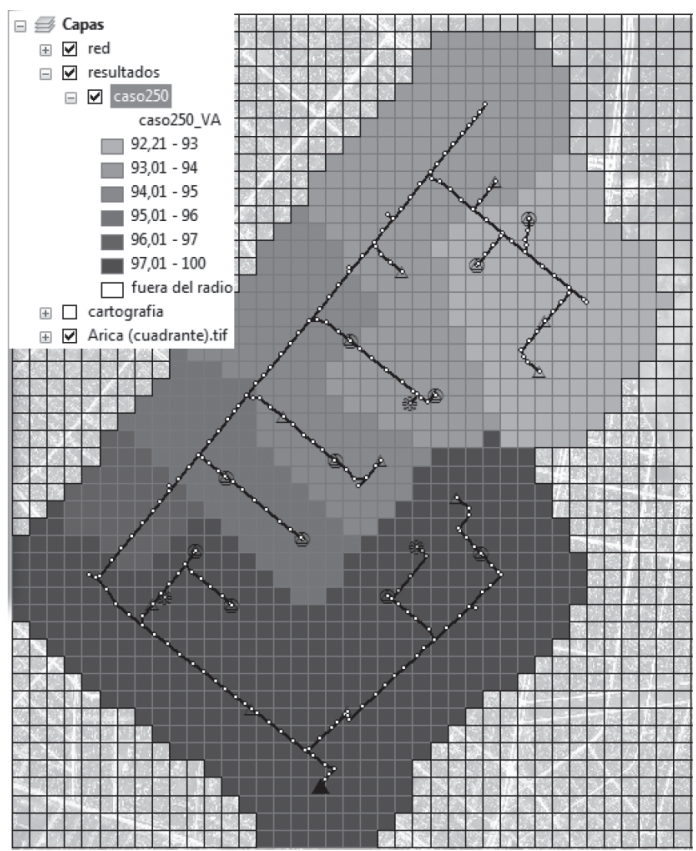

Figura 11. Grilla de valores de voltajes para el "caso250" generada de la herramienta "Crea grilla según valores de voltaje". 
Los cuadros en que la grilla no presenta color se debe a que dicho cuadro se encuentra fuera del radio de $200 \mathrm{~m}$ establecido en la Figura 8. Justamente, a este cuadro se asignó la etiqueta "fuera del radio de búsqueda", de manera que sea identificada en la leyenda vinculada a la capa.

Similar al caso anterior (Figura 10), en la Figura 11 los valores más bajos de voltaje corresponden a los puntos más alejados de la subestación.

Otros tipos de análisis [7] que es posible de realizar, por nombrar algunos, son: (1) creación de consultas, (2) búsqueda e identificación de un elemento de la red, (3) representar información según categorías y según cantidades, (4) acceso a documentos asociados a algún elemento de la red.

\section{CONCLUSIONES}

Aunque esta investigación documenta una integración a pequeña escala, queda de manifiesto la capacidad de los GIS para iniciar una completa integración de la información de servicios eléctricos. Asimismo, en un nivel básico, se observa que la incorporación de un componente espacial para el análisis de sistemas de potencia, amplía la capacidad de explotación en nuevas áreas.

Compartir la información es fundamental para la eficiencia de una organización. El aumento de la competitividad y el avance de las tecnologías de la información hacen cada vez más valioso el manejo de datos, como un recurso corporativo.

La representación de la red eléctrica por medio de la tecnología GIS proporciona el análisis visual y la presentación de información diversa, en forma sintética, ayudando así a los involucrados en la evaluación y pronta acción en la toma de decisiones.

La visualización espacial de la caída de tensión demuestra que es posible utilizar la ubicación geográfica de los elementos de la red para descubrir nuevas alternativas de soluciones en la planeación de los sistemas eléctricos.

\section{AGRADECIMIENTOS}

Los autores agradecen el apoyo de la Universidad de Tarapacá, Proyecto Mayor-UTA № 8723-06.

\section{REFERENCIAS}

[1] V. Álvarez, C. Zea y V. Zúñiga. "Sistema de información geográfico aplicado a la explotación de la infraestructura eléctrica de líneas de transmisión y subestaciones". Tesis para optar al grado de Ingeniero. Escuela Superior Politécnica del Litoral. Guayaquil, Ecuador. 2002.

[2] S.E. Collier. "Integrating GIS with circuit modeling for electric distribution systems". Milsoft Utility Solutions, p. 7. Texas, Estados Unidos. 2008. Fecha de Consulta: 11 de mayo de 2013. URL: http://milsoft.com/ downloads/presentationp/

[3] DORC. "DS 327.97 Reglamento de la ley general de servicios eléctricos". Diario Oficial República de Chile. 1998.

[4] Emel. "Manual usuario funcionalidad y modo utilización SIPRE". Documento interno empresas Emel S.A. Chile. 2000.

[5] Emel. "POLOPE001 Planificación y Desarrollo sistema Eléctrico de Emel". Documento interno empresas Emel S.A. Chile. 2004.

[6] Emel. "PROCOPE073 Proyección de la Demanda". Documento interno empresas Emel S.A. Chile. 2004.

[7] Esri. "Ayuda de ArcGIS Desktop 10". Estados Unidos. Noviembre 2010. URL:

http://help.arcgis.com/es/arcgisdesktop/10.0/ help/index.html\#//00r90000001n000000

[8] Esri. "SIG para empresas de servicios públicos y telecomunicaciones". 2001.

[9] M. Estrada. "Integración de sistemas de información geográfico de una red de distribución eléctrica”. Memoria para optar al título de Ingeniero Civil Eléctrico. Universidad de Tarapacá. Arica, Chile. 2012.

[10] V. Flores, E. Tong y G. Veintimilla. "Sistema de información geográfico para una empresa eléctrica". Tesis para optar al título de Ingeniero. Escuela Superior Politécnica del Litoral. Guayaquil, Ecuador. 1998.

[11] P. Hartley. "Electrical distribution modeling: an integration of engineering analysis and geographic information system". Tesis para optar grado de Magíster en Ciencias. Virginia Polytechnic Institute and State University. Blacksburg, Virginia, Estados Unidos. 2005.

[12] S. Martínez. "Preguntas y respuestas de ingeniería eléctrica”. Cap. 5, pp. 104-105. 
Septiembre 2007. Fecha de Consulta: 11 de mayo de 2013. URL: http://www.elistas.net/ lista/electric/

[13] J.I. Monroy. "SIG en servicios públicos". pp. 6-33. Agosto 2011. Fecha de consulta: 11 de mayo de 2013. URL: http:// www.procalculoprosis.com/ccu2201/ Seminario\%20industria\%20servicser\%20 publicos26agos.pdf

[14] O. Saheed and U. Rashidat. "Electricity distribution engineering and geographic information system (DeGIS)". Shape the change XXIII FIG congress. Munich, Germany. October, 2006. Date of visit: May 11, 2013. URL: http://www.fig.net/ pub/fig2006/papers/ts72/ts72_04_olaniyi_ usman_0591.pdf

[15] T.J. Terrell. "Building a geographic information system". IEEE Computer Applications in Power. Vol. 4, Issue 3, pp. 50-54. July, 1991. ISSN: 0895-0156.
[16] L.V. Trussell. "GIS based distribution simulation and analysis". 16th International Conference and Exhibition on Electricity Distribution-CIRED 2001. Amsterdam, The Netherlands. June 2001. Paper $N^{\circ} 5.30$. ISSN: 0537-9989.

[17] Z. Wang, D.E. Julian, M. Bass and W. Peterson. "Interpreting GIS data for operation and control of distribution networks". Power Systems Conference and Exposition. IEEE PES. Vol. 2, pp. 907-912. 2004. October 10-13, 2004.

[18] Yen-Ting Chao, Shen-Ta Lee and Hong-Chan Chang. "Application of automated mapping system to distribution transformer load management". Transmission and Distribution Conference and Exhibition 2002: Asia Pacific. IEEE/PES, Vol. 2, pp. 1179-1184. October 6-10, 2002. 